\title{
The Rho-linked mental retardation protein oligophrenin-1 controls synapse maturation and plasticity by stabilizing AMPA receptors
}

\author{
Nael Nadif Kasri, Akiko Nakano-Kobayashi, Roberto Malinow, ${ }^{1}$ Bo Li, and Linda Van Aelst ${ }^{2}$ \\ Cold Spring Harbor Laboratory, Cold Spring Harbor, New York 11724, USA
}

\begin{abstract}
Oligophrenin-1 (OPHN1) encodes a Rho-GTPase-activating protein (Rho-GAP) whose loss of function has been associated with X-linked mental retardation (MR). The pathophysiological role of OPHN1, however, remains poorly understood. Here we show that OPHN1 through its Rho-GAP activity plays a critical role in the activitydependent maturation and plasticity of excitatory synapses by controlling their structural and functional stability. Synaptic activity through NMDA receptor activation drives OPHN1 into dendritic spines, where it forms a complex with AMPA receptors, and selectively enhances AMPA-receptor-mediated synaptic transmission and spine size by stabilizing synaptic AMPA receptors. Consequently, decreased or defective OPHN1 signaling prevents glutamatergic synapse maturation and causes loss of synaptic structure, function, and plasticity. These results imply that normal activity-driven glutamatergic synapse development is impaired by perturbation of OPHN1 function. Thus, our findings link genetic deficits in OPHN1 to glutamatergic dysfunction and suggest that defects in early circuitry development are an important contributory factor to this form of MR.
\end{abstract}

[Keywords: Rho GTPase-activating protein; Oligophrenin-1; glutamatergic function; AMPA receptor; actin cytoskeleton; mental retardation]

Supplemental material is available at http://www.genesdev.org.

Received January 21, 2009; revised version accepted April 23, 2009.

The formation, maturation, and activity-dependent modification of the efficacy of glutamatergic synapses play important roles in the development of neural circuits and are critical for proper brain functions, such as information processing and storage (Li and Sheng 2003; McAllister 2007; Citri and Malenka 2008). One key mechanism for the regulation of synaptic strength, during both synaptic development and plasticity, is the synaptic incorporation and removal of AMPA-type glutamate receptors, the principal receptors for fast excitatory neurotransmission in the mammalian central nervous system (Shepherd and Huganir 2007; Citri and Malenka 2008). In concurrence with an important role for these processes in learning, memory, and cognition, accumulating evidence suggests that impairments in synaptogenesis and/or synaptic plasticity contribute to numerous mental and neurological disorders, including mental retardation (MR)

\footnotetext{
${ }^{1}$ Present address: University of California at San Diego, CMG 0634, 9500 Gilman Drive, La Jolla, CA 92093, USA.

${ }^{2}$ Corresponding author.

E-MAIL vanaelst@cshl.edu; FAX (516) 367-8815.

Article is online at http://www.genesdev.org/cgi/doi/10.1101/gad.1783809.
}

(Bagni and Greenough 2005; Chahrour and Zoghbi 2007; Vaillend et al. 2008).

MR is generally defined as a global reduction in cognitive abilities that manifests before the age of 18 (Chelly et al. 2006). The causes of MR are extremely heterogeneous, including environmental factors as well as genetic changes, such as chromosomal abnormalities and singlegene mutations (Chelly et al. 2006; Vaillend et al. 2008). Great progress has been made over recent years toward the identification of MR genes, resulting in a list of more than 280 genes (Inlow and Restifo 2004). A largely remaining challenge, however, is to connect the genetic causes of MR to processes that establish and/or modify neuronal circuit function.

Several of the currently identified genes associated with MR code for regulators and effectors of the Rho subfamily of GTP-binding proteins (van Galen and Ramakers 2005; Nadif Kasri and Van Aelst 2008), which cycle between an inactive GDP-bound state and an active GTPbound state. Members of this family are key regulators of the actin cytoskeleton and affect many aspects of neuronal development (Govek et al. 2005). Their activity is tightly controlled by dedicated guanine nucleotide exchange factors (GEFs), which promote GTP-loading, and 
GTPase-activating proteins (GAPs), which enhance hydrolysis of the bound GTP (Govek et al. 2005). Significantly, mutations in genes encoding GAPs and GEFs, as well as effectors of Rho GTPases, have been found to underlie various forms of MR (Nadif Kasri and Van Aelst 2008).

OPHN1, which encodes a Rho-GAP, was the first identified Rho-linked MR gene (Billuart et al. 1998). It was initially identified by the analysis of a balanced translocation $\mathrm{t}\left(\mathrm{X}_{;} 12\right)$ observed in a female patient with mild MR (Bienvenu et al. 1997). Subsequent studies have revealed the presence of OPHN1 mutations in families with MR associated with cerebellar hypoplasia and lateral ventricle enlargement (Tentler et al. 1999; Bergmann et al. 2003; Philip et al. 2003; des Portes et al. 2004; Zanni et al. 2005). All OPHN1 mutations identified to date have been shown, or predicted, to result in OPHN1 loss of function (Zanni et al. 2005), and interestingly, inactivation of ophn1 in mice has recently been demonstrated to recapitulate some of the human phenotypes, such as behavioral, social, and cognitive impairments (Khelfaoui et al. 2007).

The OPHN1 protein is highly expressed in the brain, where it is found in neurons of all major regions, including hippocampus and cortex, and it is present in both the axon and dendrites of principal neurons (Govek et al. 2004). To date, however, the neuronal function of OPHN1 is largely elusive. Studies from our laboratory and others have implicated OPHN1 in the regulation of spine morphology of CA1 hippocampal neurons (Govek et al. 2004; Khelfaoui et al. 2007), although it remains unclear whether OPHN1 controls spine formation or maintenance. Furthermore, the role of OPHN1 in the development or function of glutamatergic synapses remains to be elucidated.

Here, by temporally and spatially manipulating OPHN1 gene expression, we demonstrate that post-synaptic OPHN1 plays a key role in activity-dependent maturation and plasticity of excitatory synapses by regulating their structural and functional stability. Furthermore, we show that OPHN1's localization and function in excitatory synapses is dependent on synaptic activity and NMDA receptor activation. Importantly, OPHN1 regulates synaptic structure and function in a Rho-GAP activity-dependent manner by controlling the stabilization of AMPA receptors. Therefore, defective OPHN1 signaling results in destabilization of synaptic AMPA receptors and spine structure, leading to impairment in plasticity and eventually loss of spines and NMDA receptors. Together, our results indicate that critical levels of OPHN1 are necessary for proper activity-driven glutamatergic synapse development and suggest a cellular mechanism by which mutations in OPHN1 can contribute to the cognitive deficits observed in OPHN1 patients.

\section{Results}

OPHN1 regulates excitatory synaptic transmission at hippocampal CA3-CA1 synapse

To assess whether post-synaptic OPHN1 is important for synaptic function, we first examined the effects of
OPHN1 overexpression on synaptic transmission at the hippocampal CA3-CA1 pathway. CA1 neurons in cultured hippocampal slices were infected with a lentivirus coexpressing wild-type OPHN1 (OPHN1-WT) and EGFP (as a marker for infected cells) at $1 \mathrm{~d}$ in vitro (DIV). Seven days post-infection, simultaneous recordings of evoked excitatory post-synaptic currents (eEPSCs) from a CA1 pyramidal neuron overexpressing OPHN1-WT and an adjacent noninfected neuron were performed. Overexpression of OPHN1-WT resulted in a potentiation of AMPA receptor (AMPAR)-mediated transmission, but not NMDA receptor (NMDAR)-mediated transmission (Fig. 1A), indicating that ectopically expressed OPHN1 is sufficient to enhance AMPAR-mediated transmission.

We next examined the function of endogenous OPHN1 by probing the effects of reduced OPHN1 expression on synaptic transmission. To selectively reduce endogenous OPHN1 protein levels in CA1 neurons, we used RNAi. Lentiviral constructs were generated that coexpress EGFP and shRNAs targeting either the translated (OPHN1\#1) or the $3^{\prime}$-untranslated region (UTR, OPHN1\#2) of rat OPHN1 mRNA. Both OPHN1\#1 and OPHN1\#2 shRNA drastically reduced endogenous OPHN1 protein levels in hippocampal neurons, whereas a control shRNA (scr\#1) was ineffective (Supplemental Fig. S1).

CA1 neurons in hippocampal slices were then infected with one of the above lentiviruses at $1 \mathrm{DIV}$ and recorded at 8 DIV. OPHN1\#1 as well as OPHN1\#2 shRNA significantly depressed both AMPAR- and NMDAR-mediated transmission (Fig. 1B,C). In contrast, the control scr\#1 shRNA did not affect AMPAR- or NMDAR-mediated currents (Fig. 1D). To further test the specificity of OPHN1 RNAi, we performed rescue experiments using OPHN1 cDNA that lacks the 3 '-UTR and is therefore resistant to OPHN1\#2 shRNA-mediated RNAi. Neurons were infected with a lentiviral vector that coexpresses OPHN1\#2 shRNA and RNAi-resistant OPHN1-WTEGFP. The levels of OPHN1-WT-EGFP in these neurons were nearly similar to those of endogenous OPHN1 in control cells (Supplemental Fig. S2A,B). Most importantly, these neurons did not show any defects in AMPAR- or NMDAR-mediated transmission (Fig. 1E), indicating that the observed effects of OPHN1 RNAi are specific. Thus, endogenous OPHN1 function is required for both AMPAR- and NMDAR-mediated transmission.

To determine whether OPHN1's Rho-GAP activity, which represses the RhoA/Rho-kinase pathway in hippocampal neurons (Supplemental Fig. S3; Govek et al. 2004), is important for its function in synaptic transmission, we generated an OPHN1 mutant, OPHN1-GAP, which virtually lacks Rho-GAP activity (Supplemental Fig. S4). This mutant was then tested for its ability to restore the defects in AMPAR- and NMDAR-mediated transmission of OPHN1\#2 shRNA-expressing neurons. In contrast to OPHN1-WT, coexpression of RNAi-resistant OPHN1GAP failed to rescue the OPHN1\#2 shRNA-evoked defects (Fig. 1F). OPHN1-GAP and OPHN1-WT were, however, expressed at similar levels and displayed a similar subcellular distribution (Supplemental Fig. S2). These results indicate that OPHN1's Rho-GAP activity is 
A
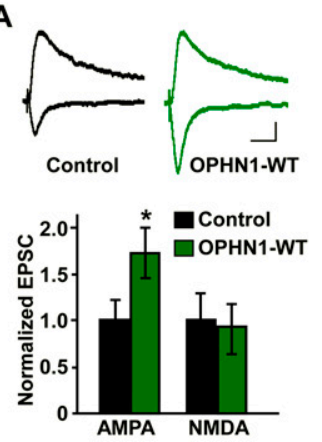

D

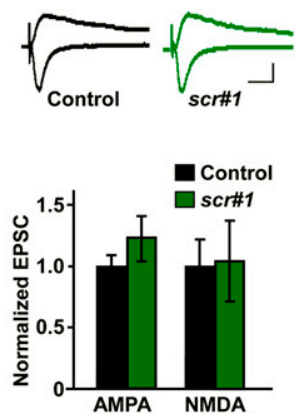

B

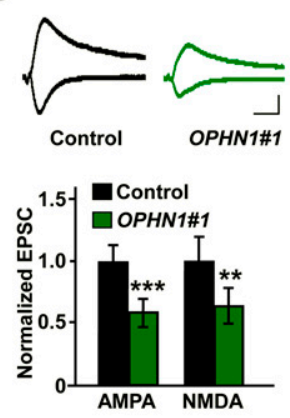

E
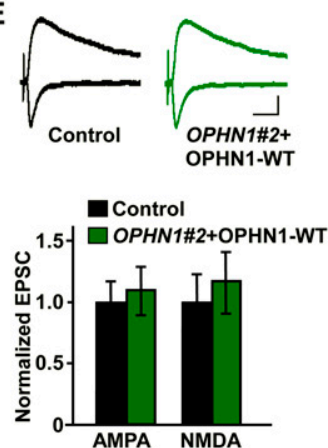

C
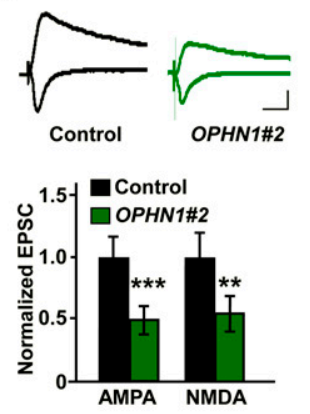

F
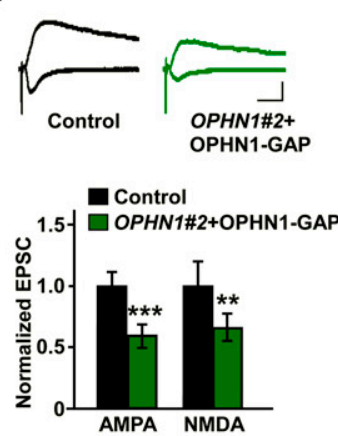

Figure 1. Post-synaptic OPHN1 regulates synaptic transmission. ( $A-F$, top panels) Representative traces of EPSCs recorded simultaneously from a pair of uninfected neurons (control) and neurons infected with indicated lentiviruses. EPSCs from both -60 - and $+40-\mathrm{mV}$ holding potentials are shown. Bars, $20 \mathrm{msec}$ and $20 \mathrm{pA}$. (Bottom panels) Quantification of the EPSCs mediated by both AMPAR and NMDAR for each experimental condition as indicated. The EPSC amplitude was normalized to the mean value of EPSCs from control neurons. (A) OPHN1-WT: $n=12$. (B) OPHN1\#1: $n=$ 10. $(C)$ OPHN1\#2: $n=12$. $(D) s c r \# 1: n=8 .(E)$ OPHN1\#2 + OPHN1-WT: $n=12$ pairs. $(F)$ OPHN1\#2+OPHN1-GAP $n=11$. Data are shown as mean \pm SEM. $\left(^{\star}\right) P<0.05 ;\left(^{\star \star}\right) P<$ $0.01 ;\left(^{\star \star \star}\right) P<0.005$ by paired Student's $t$-test. required for normal synaptic transmission at the CA3CAl synapse. Consistent with this, ectopic expression of OPHN1-GAP, in contrast to OPHN1-WT, failed to potentiate AMPAR-mediated transmission (Supplemental Fig. S5).

\section{OPHN1 regulates glutamatergic synapse maturation}

Since OPHN1-linked MR has been associated with developmental defects, we postulated that OPHN1 might influence glutamatergic synaptic transmission by controlling synaptic maturation that occurs during the period that constructs are expressed. To test this, we examined the effect of OPHN1 knockdown on miniature EPSCs (mEPSCs), the responses to spontaneous release events at single synapses, of CA1 pyramidal cells. Hippocampal slices were infected with lentivirus expressing OPHN1\#1 or OPHN1\#2 shRNA at $1 \mathrm{DIV}$, and the frequency and amplitude of mEPSCs were measured at 4 DIV and 8 DIV (Fig. 2A-C). Normally, there is a significant increase in mEPSC frequency and a small to no increase in mEPSC amplitude, during early development of the CA3-CA1 synapse (Fig. 2A-C, control: 4 vs. 8 DIV; Barria and Malinow 2005; Li et al. 2007). Strikingly, both OPHN1\#1 and OPHN1\#2 shRNA largely prevented the developmental increase in mEPSC frequency (Fig. 2B) without significantly influencing mEPSC amplitude (Fig. 2C). Of note, the efficiency of OPHN1 knockdown was similar at 4 DIV and 8 DIV (Fig. 2G). A change in frequency usually reflects a change in the number of synaptic sites or presynaptic release probability. We observed that OPHN1 RNAi in post-synaptic cells did not affect paired-pulse facilitation (PPF) (Supplemental Fig. S6), a measurement of presynaptic release. Thus, our data indicate that post-synaptic OPHN1 regulates excitatory synaptic maturation by controlling the number of functional, AMPAR-containing, synapses. We also examined the impact of OPHN1 knockdown on inhibitory (GABAergic) synapse maturation in CA1 pyramidal cells. We found that OPHN1 knockdown during development did not influence the frequency or amplitude of GABA miniature inhibitory post-synaptic currents (mIPSCs) (Fig. 2D-F). Together, our findings indicate that OPHN1 is critical for excitatory, but not inhibitory, synapse maturation and that the effect of OPHN1 knockdown on synaptic maturation occurs in a cell-autonomous manner.

\section{Activity-dependent regulation of OPHN1 function and localization in excitatory synapses}

Because knockdown of OPHN1 interferes with glutamatergic synapse maturation that is normally driven by spontaneous activity and NMDAR activation (Zhu et al. 2002; Barria and Malinow 2005), we reasoned that activity blockade should occlude the effects of OPHN1 knockdown. To test this, we incubated organotypic hippocampal slices in medium containing tetrodotoxin (TTX), or high concentration of $\mathrm{MgCl}_{2}$, and then evaluated the effects of OPHN1 knockdown on synaptic transmission in the CA3-CA1 synapse. TTX or highconcentration $\mathrm{MgCl}_{2}$ treatment occluded the depression effect of OPHN1 knockdown on both AMPAR- and NMDAR-mediated transmission (Fig. 3A), indicating that OPHN1 participates in activity-driven increase in glutamatergic function. 
A

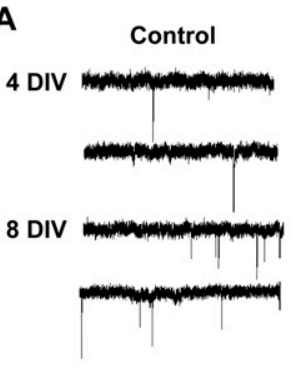

D

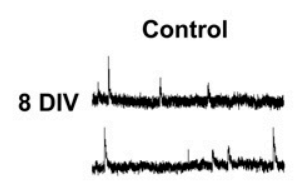

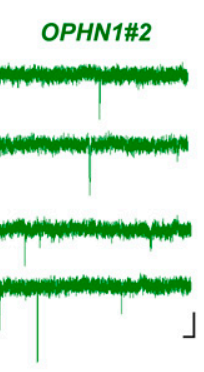

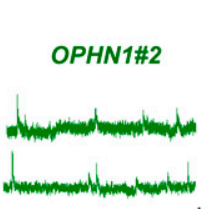

B

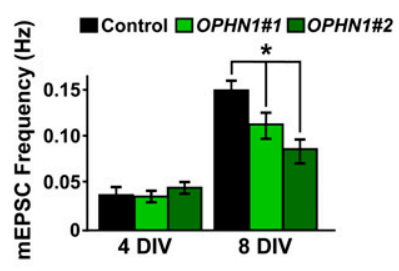

C

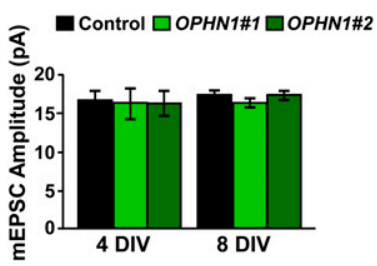

$\mathbf{E}$

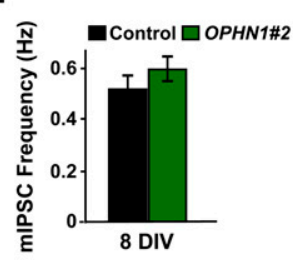

$\mathbf{F}$

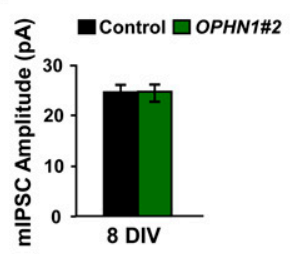

G

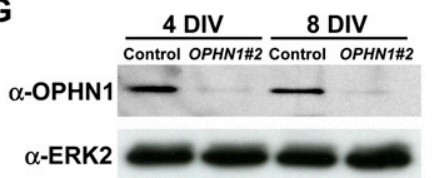

Figure 2. OPHN1 is critical for normal synaptic maturation. (A) Representative traces of excitatory miniature events recorded at -60 $\mathrm{mV}$ from uninfected neurons (control) and neurons expressing OPHN1\#2 shRNA at different developmental stages (4 and 8 DIV). Bars, 2 sec and 20 pA. $(B, C)$ Quantification of mEPSC frequency $(B)$ and amplitude $(C)$ for control and OPHN1\#1 and OPHN1\#2 shRNAexpressing neurons at different developmental stages. $(B)$ Four days in vitro: $n=18$ cells for all groups; 8 DIV: control: $n=30$ cells; OPHN1\#1: $n=21$ cells; OPHN1\#2: $n=30$ cells. $(C)$ Four days in vitro: $n=18$ cells for all groups, 8 DIV: control: $n=30$ cells; OPHN1\#1: $n=21$ cells; OPHN1\#2: $n=30$ cells. $(D)$ Representative traces of inhibitory miniature events recorded at $+10 \mathrm{mV}$ from uninfected neurons (control) and neurons expressing OPHN1\#2 shRNA at 8 DIV. Bars, 1 sec and $20 \mathrm{pA}$. $(E, F)$ Quantification of mIPSC frequency (E) and amplitude $(F)$ from control and OPHN1\#2 shRNA-expressing neurons at 8 DIV ( $n=24$ for both groups). Data are shown as mean \pm SEM. $\left(^{*}\right) P<0.005$ by Student's $t$-test. $(G)$ Immunoblot of extracts prepared from hippocampal neurons 4 and $8 \mathrm{~d}$ post-infection with scr\#1 (control) or OPHN1\#2 shRNA probed with anti-OPHN1 and anti-ERK2 antibody as a loading control.

To further assess whether the function of OPHN1 in the glutamatergic synapse depends on neuronal activity, we examined whether treatment of slices with TTX, or high concentration of $\mathrm{MgCl}_{2}$, prevents the potentiation effect of ectopically expressed OPHN1 on AMPARmediated transmission. We found that this is, indeed, the case (Fig. 3B). Significantly, the NMDAR antagonist 2-amino-5-phosphonovaleric acid (APV) also blocked the potentiation effect of OPHN1 (Fig. 3B), indicating that NMDAR activation is required for OPHN1 synaptic function.

Since TTX, $\mathrm{MgCl}_{2}$, or APV treatment did not affect the global expression levels of endogenously or ectopically expressed OPHN1 (Supplemental Fig. S7), we hypothesized that normal spontaneous neuronal activity regulates OPHN1's synaptic distribution, thereby controlling OPHN1 synaptic function. To test this, we tagged OPHN1 at its C terminus with EGFP and introduced OPHN1EGFP together with a red fluorescent protein, tDimer, as a cellular marker, into CA1 cells in organotypic hippocampal slices by biolistic transfection. The subcellular distribution of OPHN1 was examined by dual-channel two-photon laser scanning microscopy (TPLSM). We found that OPHN1-EGFP is more than twofold enriched in the spines compared with dendritic shafts when slices were maintained in normal media (Fig. 3C-F). However, maintaining slices in TTX, or high concentration of $\mathrm{MgCl}_{2}$, significantly reduced the enrichment of OPHN1 in spines (Fig. 3D-F). Also, maintaining slices in APV largely prevented OPHN1 enrichment in spines (Fig. $3 \mathrm{D}-\mathrm{F})$, indicating that OPHN1 recruitment in spines is dependent on NMDAR activation. Notably, a similar activity-dependent redistribution was observed for OPHN1-GAP (Supplemental Fig. S2C-E), indicating that OPHN1's Rho-GAP activity is not required for its recruitment and that other domain(s) in OPHN1 and/or interactions are involved. With regard to this, we found that an OPHN1 mutant lacking the N-terminally located $\mathrm{PH}$ domain is absent in spines, and, in fact, only detectable in the nucleus (data not shown), implying that the $\mathrm{PH}$ domain is important for OPHN1's localization.

Together, our results support a model in which OPHN1 is recruited or stabilized in dendritic spines by spontaneous synaptic activity through the activation of NMDARs. This activity-dependent regulation of OPHN1 is critical for its role in controlling the functional maturation of excitatory synapses and facilitating the strengthening of synaptic transmission during synaptic development. 
A
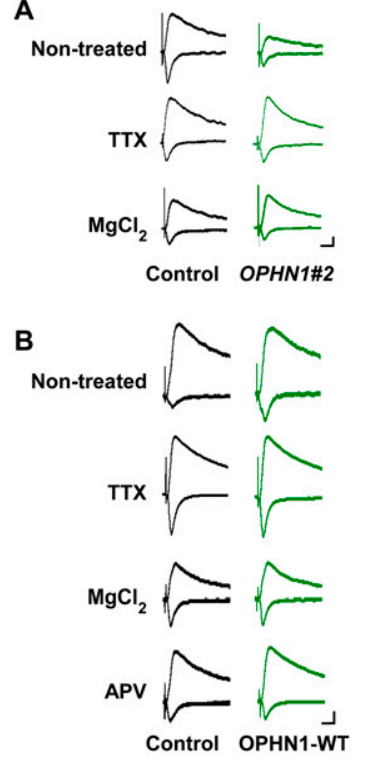

C

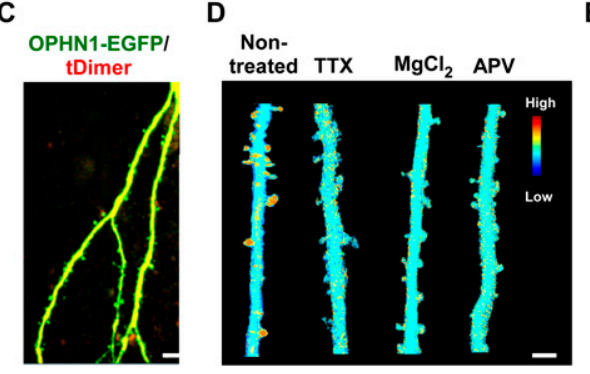

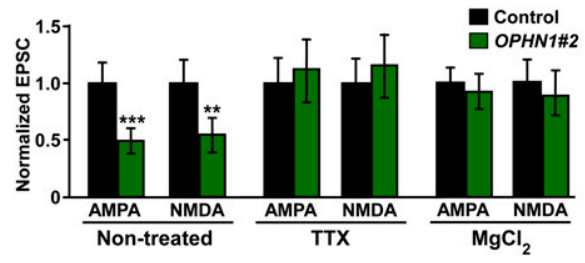

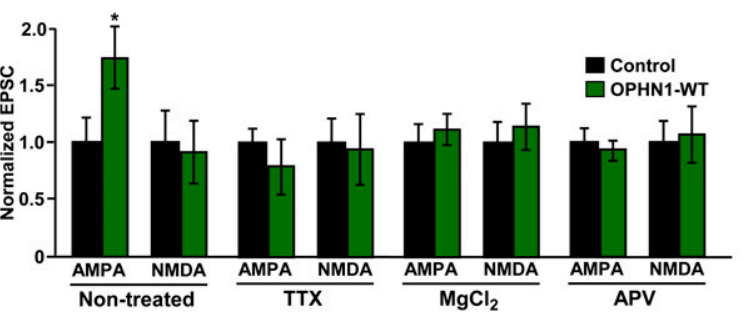

E

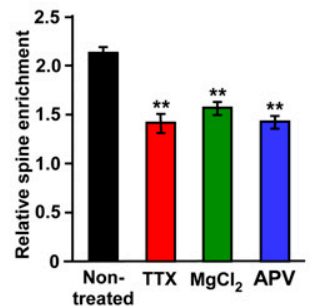

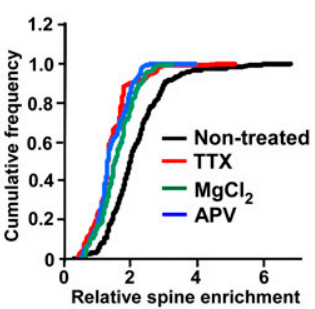

Figure 3. Spontaneous neuronal activity is important for OPHN1's synaptic function and localization. (A, left panel) Representative traces of EPSCs recorded simultaneously from a pair of uninfected neurons (control) and OPHN1\#2 shRNA-expressing neurons under the indicated conditions. (Right panel) Quantification of the EPSCs mediated by both AMPAR and NMDAR for each experimental condition as indicated (untreated: $n=12$ pairs; TTX: $n=10$ pairs; $\mathrm{MgCl}_{2}: n=11$ pairs). Data are shown as mean \pm SEM. (**) $P<0.01$; $\left({ }^{\star \star \star}\right) P<0.005$, by paired Student's $t$-test. $(B$, left panel) Representative traces of EPSCs recorded simultaneously from a pair of uninfected neurons (control) and OPHN1-WT-expressing neurons under the indicated conditions. (Right panel) Quantification of the EPSCs mediated by both AMPAR and NMDAR for each experimental condition as indicated (untreated: $n=12$ pairs; TTX-treated: $n=$ 12 pairs; $\mathrm{MgCl}_{2}$-treated: $n=10$ pairs; APV-treated: $n=9$ pairs). Data are shown as mean \pm SEM. $\left({ }^{\star}\right) P<0.05$ by paired Student's $t$-test. $(C)$ Representative images of dendritic branches of a hippocampal CA1 pyramidal neuron cotransfected with OPHN1-EGFP (green) and tDimer (red) maintained in normal media acquired with TPLSM. Bar, $10 \mu \mathrm{m}$. $(D)$ Ratio images of cells expressing OPHN1-EGFP and tDimer are shown under the indicated conditions. Blue depicts low OPHN1 density, and red depicts high density. Bar, $5 \mu \mathrm{m}$. $(E)$ Quantification of enrichment of OPHN1-EGFP in spines for each experimental condition as indicated (untreated: $n=7$ cells; TTXtreated: $n=8$ cells; $\mathrm{MgCl}_{2}$-treated: $n=7$ cells; APV-treated: $n=6$ cells). $(F)$ Cumulative distributions of enrichment of OPHN1-EGFP in spines from the data plotted in $E$. Data are shown as mean \pm SEM. $\left(^{\star \star}\right) P<0.01$ by the Kolmogorov-Smirnov test.

\section{OPHN1 is critical for stabilizing and maintaining spine structure}

Since OPHN1 is critical for the functional maturation of synapses, we speculated that it might also be important for their structural maturation, which involves growth and stabilization of newly formed and existing spines. We observed that acute knockdown of OPHN1-i.e., over a period of 2 to $3 \mathrm{~d}$-reduces spine length/size without affecting spine density (Supplemental Fig. S8; Govek et al. 2004). Consistently, our electrophysiological data showed that OPHN1 knockdown for $3 \mathrm{~d}$ has no effect on mEPSC frequency (Fig. 2A,B, 4 DIV). However, knockdown of OPHN1 during an extended period (for $7 \mathrm{~d}$ ) led to a re- duction in mEPSC frequency (Fig. 2A,B, 8 DIV), which can be explained by a reduction in the number of synapses. These findings suggested to us that OPHN1 is likely required for the stabilization and growth of existing synapses, rather than synaptogenesis.

If this is the case, knockdown of OPHN1 during an extended period should eventually result in a reduction in spine density, whereas ectopic expression of OPHN1 should not affect spine density, but increase spine size. To test this, CA1 neurons in hippocampal slices were infected with lentivirus coexpressing EGFP and OPHN1\#2 shRNA, scr\#1 shRNA, or no shRNA (control vector) at 1 DIV and imaged with TPLSM at 8 DIV. Spine density and size on apical dendrites were measured. OPHN1\#2 
shRNA, but not scr\#1 shRNA, significantly reduced spine density at 8 DIV when compared with control vector (Fig. 4A,B). OPHN1\#2 shRNA also significantly decreased spine size, although to a lesser extent (Fig. 4C). Importantly, coexpression of OPHN1-WT, but not OPHN1GAP, rescued the OPHN1\#2 shRNA-evoked spine density and spine size phenotypes (Fig. 4A-C), implying that OPHN1's Rho-GAP activity is required for stabilizing/ maintaining spine structure. Consistent with this, elevated and prolonged RhoA activity has been coupled to reduced spine length, size, and density (Nakayama et al. 2000; Tashiro et al. 2000; Ryan et al. 2005; Sfakianos et al. 2007; Kang et al. 2009). Conversely, we found that overexpression of OPHN1 did not alter spine density but significantly increased spine size at 8 DIV (Fig. 4D-F). No increase in spine size was seen when OPHN1-GAP was ectopically expressed (Supplemental Fig. S9). These data support the idea that OPHN1/RhoA-mediated signaling is not critical for synaptogenesis, but rather stabilization and growth of the existing synapses.

To substantiate OPHN1's involvement in spine stabilization/maintenance, we determined the number of transient spines (lifetime $<1 \mathrm{~h}$ ) and of spine additions and losses, the turnover rate (TOR), in neurons with and without knockdown of OPHN1 during basal activity. Specifically, CA1 neurons in hippocampal slices were biolistically cotransfected with an OPHN1\#2 or scr\#1 shRNA-expressing construct and a tDimer expression vector. At 4 and 8 d post-transfection, dynamics of spines were monitored over a 2- to 3-h time period by 2-photon imaging. Whereas in scr\#1 shRNA-expressing neurons the number of transient spines and the spine TOR significantly decreased with development (Fig. 4G-I, 4 vs. $8 \mathrm{~d}$ ), in OPHN1\#2 shRNA-expressing neurons, both the number of transient spines and spine TOR remained elevated (Fig. 4G-I). These data imply that OPHN1 knockdown decreases spine stability during spontaneous activity, which could underlie the observed arrest of excitatory synaptic maturation.

Together, our findings strongly suggest that OPHN1/ RhoA-mediated signaling is critical for the stabilization and maintenance of existing synapses, but is not sufficient to promote new synapse formation.

\section{OPHN1 is critical for structural and functional synaptic plasticity}

Since OPHN1 regulates spontaneous activity-dependent plasticity, which shares many properties with a more acute form of plasticity, long-term potentiation (LTP) (Barria and Malinow 2005), we speculated that OPHN1 might also be important for LTP. To examine this, OPHN1-WT-EGFP or OPHN1\#2 shRNA, together with tDimer, were expressed in CA1 neurons, and the slices were incubated in elevated $\mathrm{MgCl}_{2}$ during the period (4 d) that the constructs were expressed. This normalizes NMDA current between infected and uninfected CA1 neurons, thereby precluding the effect of NMDAR
Figure 4. OPHN1 controls maintenance of spine morphology. (A) Representative images of secondary apical dendrites from CA1 pyramidal neurons infected at 1 DIV with indicated lentiviruses, acquired with TPLSM at 8 DIV. Bars, $10 \mu \mathrm{m}$. $(B, C)$ Quantification of the spine density $(B)$ and spine size $(C)$ for each experimental condition as indicated (control: $n=9$ cells; scr\#1: $n=9$ cells; OPHN1\#2: $n=9$ cells; OPHN1\#2 + OPHN1-WT: $n=8$ cells; OPHN1\#2 + OPHN1-GAP: $n=8$ cells). $(D)$ Representative images of secondary apical dendrites from CA1 pyramidal neurons infected at 1 DIV with lentivirus expressing EGFP only (control), or OPHN1-WT and EGFP, acquired with TPLSM at 8 DIV. Bars, $10 \mu \mathrm{m}$. $(E, F)$ Quantification of spine density $(E)$ and size $(F)$ for control neurons and neurons expressing OPHN1-WT $(n=8$ for all conditions). ( $G$ ) Time-lapse images of a dendrite from a CA1 hippocampal neuron coexpressing OPHN1\#2 shRNA and EGFP (at 8 d post-transfection) showing transient protrusions (arrowhead). Bar, 2 $\mu \mathrm{m} .(H, I)$ Fraction of transient spines (lifetime $<1 \mathrm{~h}$ ) $(H)$ and spine TOR $(I)$ in scr\#1 shRNA versus OPHN1\#2 shRNA-expressing neurons at 4 and 8 d post-transfection ( $n=6$ cells for all groups). Data are shown as mean \pm SEM. $\left({ }^{\star}\right) P<0.05 ;\left(^{\star \star}\right) P<0.01$ by Student's $t$-test.
A

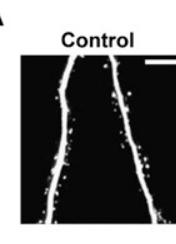

B

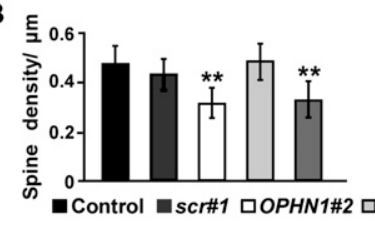

D

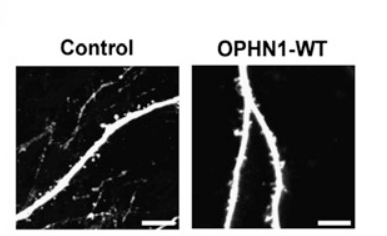

E

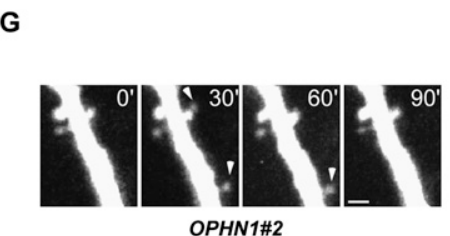

C

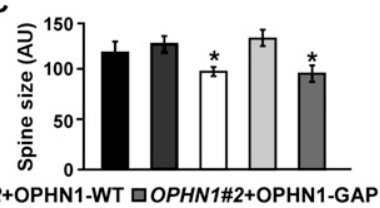

F
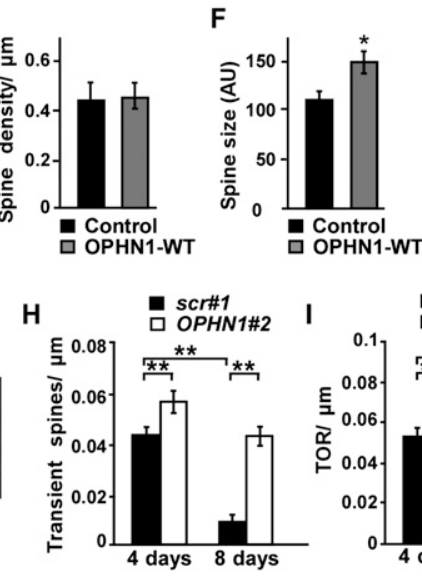

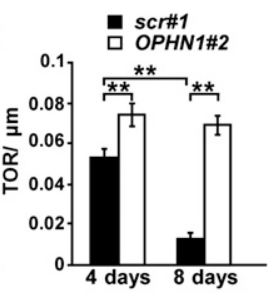


depression on synaptic plasticity (Fig. 3A,B). A chemical LTP (cLTP) protocol was used to induce synaptic plasticity (Kopec et al. 2006, 2007), and changes in individual spines were monitored by TPLSM. To ensure the efficacy of the cLTP protocol, we first tested the impact of cLTP on neurons expressing the AMPAR subunit GluR1 tagged with a $\mathrm{pH}$-sensitive form of GFP (super ecliptic pHluorin; SEP) and tDimer. Consistent with previous studies (Kopec et al. 2006), we found that cLTP, which induces transient synchronized bursting in organotypic slices
(Kopec et al. 2007), triggered a rapid and persistent increase in spine size (Fig. 5A) and in the amount of SEP-GluR1 on the spine surface in these neurons (Fig. 5A). Similarly, in neurons expressing OPHN1-EGFP, we found that cLTP triggered a rapid and persistent increase in spine size (Fig. 5B). Significantly, cLTP also induced a rapid and persistent increase in the amount of OPHN1 in spines to an extent that is similar to the increase in spine GluR1 during cLTP (Fig. 5B), further supporting the idea that neuronal activity recruits OPHN1 into spines.
A
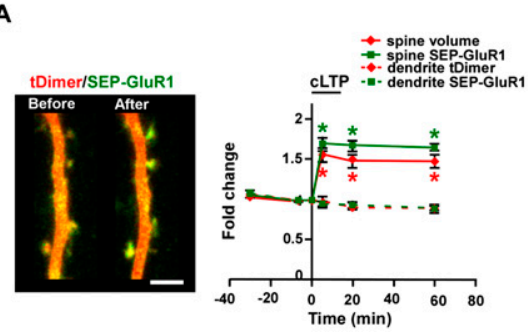

D

C
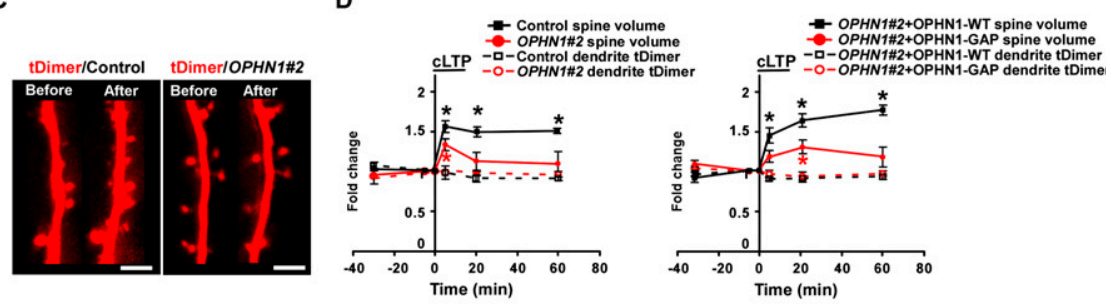

E

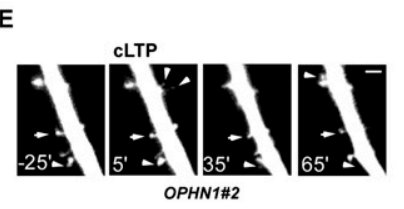

F

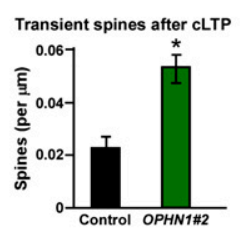

G

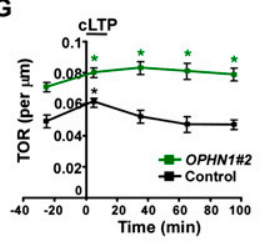

H

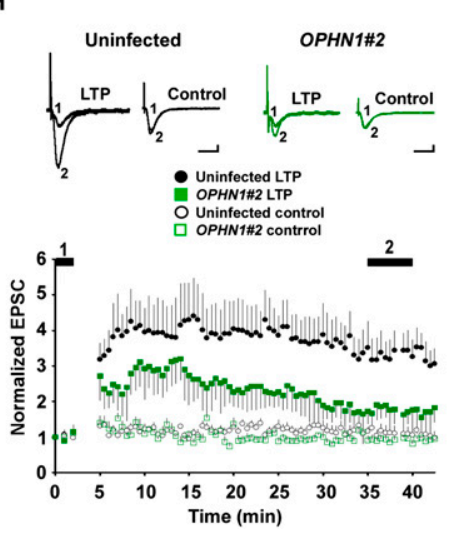

I

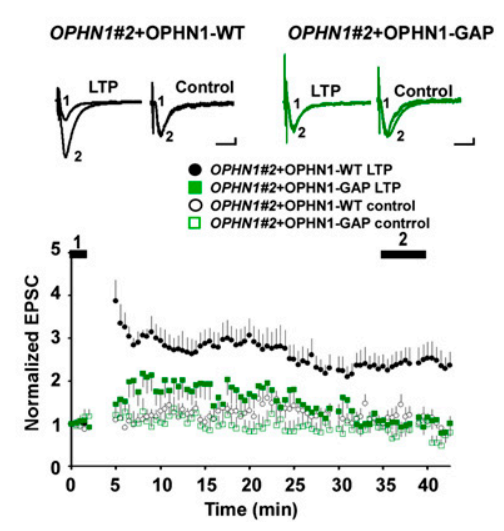

Figure 5. OPHN1 is critical for structural and functional plasticity. $(A, B$, left $)$ Images of a CA1 pyramidal cell expressing tDimer and SEP-GluR1 $(A)$ or OPHN1-EGFP $(B)$ taken at $-30 \mathrm{~min}$ (before) and $+60 \mathrm{~min}$ (after) relative to cLTP induction. Bar, 5 $\mu \mathrm{m}$. (Right) Quantification of the integrated red (volume) and green (SEP-GluR1 or OPHN1-EGFP) fluorescence for spines, as well as the mean red (tDimer) and mean green (SEP-GluR1 or OPHN1-EGFP) value for dendrites at each time point during the cLTP experiments. Each region of interest is normalized to its value at the $-5-\mathrm{min}$ time point. The black bar denotes cLTP induction ( $n=6$ cells for all groups). $(C)$ Images of a CA1 pyramidal cell expressing tDimer (control) or tDimer and OPHN1\#2 shRNA taken at $-30 \mathrm{~min}$ and $+60 \mathrm{~min}$ relative to cLTP induction. Bar, $5 \mu \mathrm{m}$. $(D)$ Quantification of the integrated red (volume) fluorescence for spines, as well as the mean red (tDimer) value for dendrites at each time point during the cLTP experiments for each experimental condition as indicated. Each region of interest is normalized to its value at the $-5 \mathrm{~min}$ time point. The black bar denotes cLTP induction ( $n=6$ cells for all groups). (E) Images of a CA1 pyramidal cell expressing OPHN1\#2 shRNA and tDimer, taken at indicated time points, showing spine size increase/decrease (arrow) andspine appearance/disappearance (arrowhead). Bar, $2 \mu \mathrm{m}$. (F) Fraction of transient spines (lifetime $<1 \mathrm{~h}$ ) after cLTP in control cells versus OPHN1\#2 shRNA-expressing neurons ( $n=6$ cells for all groups). (G) Spine TOR was measured before and after cLTP in control and OPHN1\#2 shRNA-expressing neurons at indicated time points $(n=6$ cells for all groups). Data are shown as mean \pm SEM. $\left(^{*}\right) P<0.01$ by Student's $t$-test. $(H, I)$ LTP was induced in CA1 neurons infected with indicated lentiviruses. Slices were incubated in medium contain-

ing $10 \mathrm{mM} \mathrm{MgCl}_{2}$. (Top) Representative traces of AMPAR-mediated EPSCs. Traces are averaged for time points before (1) andafter (2) LTP induction for the induced pathway (LTP) and control pathway (control). Bars, $25 \mathrm{msec}$ and 10 pA. (Bottom) Normalized AMPARmediated EPSCs before (1) and after (2) LTP induction for the induced pathway (closed symbols) andcontrol pathway (open symbols) in neurons infected with lentiviruses, as indicated. $(H)$ Control: $n=11 ;$ OPHN1\#2: $n=12$ reduced to $11 ; P<0.05$ measured between 35 and 40 min. (I) OPHN1\#2+OPHN1-WT: $n=9$; OPHN1\#2+ OPHN1-GAP: $n=10 ; P<0.05$ measured between 35 and 40 min by a Mann-Whitney test. 
The increase of OPHN1 in spines is unlikely to be a passive recruitment due to the increase in spine size, since other proteins, such as NMDARs, do not increase in spines during cLTP (Kopec et al. 2006). On the other hand, expression of OPHN1\#2 shRNA largely prevented the spine size increase induced by cLTP (Fig. 5C,D), which, importantly, could be restored by coexpression of RNAiresistant OPHN1-WT, but not OPHN1-GAP (Fig. 5D). These data indicate that OPHN1 is required for cLTPinduced spine size increase and that OPHN1's Rho-GAP activity is important for this event.

Interestingly, several observations in the cLTP experiments further suggested that spines were less stable in OPHN1 knockdown neurons. After cLTP, we observed a larger fraction of transient spines (lifetime $<1 \mathrm{~h}$ ) in OPHN1\#2 shRNA-expressing neurons than in control neurons (Fig. 5E,F). Furthermore, the turnover rate (TOR) was significantly increased after knockdown of OPHN1, both before and after cLTP (Fig. 5E,G). In OPHN1\#2 shRNA-expressing neurons, the high TOR was due to a nearly equal contribution of spine addition and loss, resulting in little change in the total number of spines. In control neurons, the TOR increased transiently after cLTP (Fig. 5G), mostly due to spine additions. In summary, knockdown of OPHN1 prevents cLTP-induced spine increase and results in a higher TOR and number of transient spines after cLTP, implying that stabilization of spine morphological changes may be impaired.

To investigate whether OPHN1 is also required for the conventional, electrically induced LTP, we examined LTP at the Schaffer collateral pathway in control and OPHN1\#2 shRNA-expressing CA1 neurons. To preclude the effect of NMDAR depression on synaptic plasticity, we incubated the brain slices in elevated $\mathrm{MgCl}_{2}$ during the period that OPHN1\#2 shRNA was expressed, which normalized NMDA current between control and OPHN1\#2 shRNA-expressing neurons (Fig. 3A). LTP-inducing stimuli produced a robust and lasting potentiation in control, uninfected, neurons (Fig. 5H). Interestingly, when LTP was induced in neurons expressing OPHN1\#2 shRNA, we only observed an initial, transient, increase in synaptic transmission, which returned to baseline levels 35-40 min after induction (Fig. 5H). These results imply that OPHN1 knockdown significantly decreases LTP. The deficit in LTP was rescued when RNAi-resistant OPHN1-WT, but not OPHN1-GAP, was coexpressed with OPHN1\#2 shRNA (Fig. 5I), indicating that OPHN1's Rho-GAP activity is important for its effect on LTP.

\section{OPHN1 controls synaptic function and structure by stabilizing AMPA receptors}

Several observations have indicated a strong positive correlation between changes in spine size and synaptic strength during LTP (Harris et al. 1992; Engert and Bonhoeffer 1999; Matsuzaki et al. 2004; Kopec et al. 2006). How changes in spine structure and synaptic strength are coordinated is currently under intense investigation. Our data implicate OPHN1 in the regulation of both structure and function of excitatory synapses and suggest that OPHN1 could be one of the factors that coordinate structural and functional changes that occur during synaptic development and plasticity.

Significantly, we found that OPHN1's Rho-GAP activity, which represses RhoA activity in neurons, is required for maintaining spine structure, enhancing AMPAR-mediated transmission, and structural and functional plasticity, thus supporting the involvement of a RhoA-mediated signaling pathway. A major downstream target of RhoA and its regulators, including OPHN1, is the actin cytoskeleton (Govek et al. 2005). Actin remodeling, which occurs in dendritic spines, drives changes in spine morphology and is required for stable LTP, and, interestingly, several recent observations pointed out that actin remodeling in the close proximity of AMPARs could be critical for stabilizing AMPARs, thereby linking synaptic function and structure (for review, see Cingolani and Goda 2008). Hence, an intriguing question is whether OPHN1, as a regulator of RhoA and the actin cytoskeleton, is involved in the stabilization of AMPARs and in that way controls and coordinates structural and functional changes.

As a first step toward addressing this question, we investigated whether OPHN1 resides in the proximity of AMPARs. In fact, given that OPHN1 translocates into spines upon synaptic activity (Fig. 3C-F), where it enhances synaptic transmission, we reasoned that OPHN1 could potentially form a complex with AMPARs at the synapse, thereby facilitating the local regulation of actin dynamics and of synaptic AMPARs. To investigate this, we performed coimmunoprecipitation experiments using synaptosomes prepared from postnatal day 14 rat brains. OPHN1 specifically coimmunoprecipitated with the AMPAR subunits, GluR2 and GluR1, and vice versa, GluR2 and GluR1 specifically coimmunoprecipitated with OPHN1 (Fig. 6A,B). Furthermore, we found that OPHN1 colocalized with both GluR1 and GluR2 in spines (Fig. 6D). On the other hand, no interaction was detected between OPHN1 and the NMDAR subunit NR1 (Fig. 6C), suggesting that OPHN1 is not a component of NMDAR complexes. Thus, OPHN1 is coupled to AMPARs at the synapse.

Next, we reasoned that if OPHN1 controls synaptic function and structure by stabilizing AMPARs, then stabilizing AMPARs should prevent the defects in synaptic transmission, and in spine density/size, caused by knockdown of OPHN1. To test this, we used a peptide (GluR2-3Y) derived from the AMPAR GluR2 C terminus, which was described previously to stabilize AMPARs in the synapse (Ahmadian et al. 2004). This peptide has been demonstrated to block regulated AMPAR endocytosis and LTD (Ahmadian et al. 2004; Brebner et al. 2005; Fox et al. 2007; Wong et al. 2007) and to rescue the defect in synaptic depression and spine loss resulting from neuregulin-1/erbB4 loss of function (Li et al. 2007). It remained, however, to be determined whether the GluR2-3Y peptide also blocks naturally occurring AMPAR endocytosis that underlies spontaneous activity-induced LTD (Zhu et al. 2002). To investigate this, we incubated hippocampal brain slices with the GluR2-3Y peptide for $4 \mathrm{~d}$ and then measured the frequency and 
A
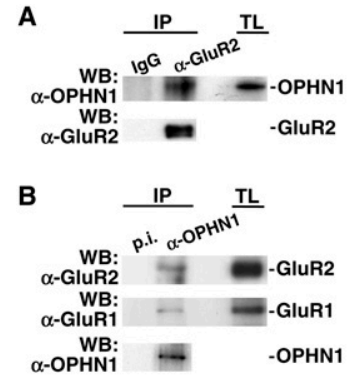

D

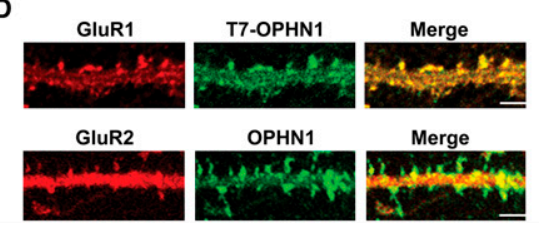

Figure 6. OPHN1 associates with AMPA receptor complexes. (A) Synaptosmal fractions prepared from rat brains were incubated with anti-GluR2 or anti-GluR1 antibody and immunoblotted with anti-OPHN1 and anti-GluR2 or anti-GluR1 antibody. Negative controls were IgG antibodies. (TL) Total lysate. $(B)$ Synaptosmal fractions were incubated with antiOPHN1 serum and immunoblotted with anti-GluR2, antiGluR1, and anti-OPHN1 antibody. Negative control was preimmune serum (p.i.). (C) Synaptosmal fractions were incubated with anti-OPHN1 serum and immunoblotted with anti-NR1 and anti-OPHN1 antibody. Negative control was preimmune serum (p.i.). (D) Colocalization of OPHN1 and AMPARs, GluR1 and GluR2, in dendritic spines. Cultured hippocampal neurons expressing T7-OPHN1 (top panel) or OPHN1 (bottom panel) were immunostained at 20 DIV with anti-mouse $\mathrm{T} 7$ and antirabbit GluR1 (top panel) or anti-rabbit OPHN1 and anti-mouse GluR2 (bottom panel) antibodies in membrane-permeabilizing conditions. Bars, $5 \mu \mathrm{m}$.

amplitude of mEPSCs. The GluR2-3Y peptide, but not the control GluR2-3A peptide, significantly increased mEPSC frequency (Fig. 7A,B). No change in amplitude was observed (Fig. 7C). These data imply that the GluR2$3 \mathrm{Y}$ peptide blocks AMPAR endocytosis that is driven by spontaneous activity. Consistent with this, we found that neurons incubated for $4 \mathrm{~d}$ with the GluR2-3Y peptide displayed an increase in spine density, a small enlargement in spine size, and an increase in cell-surface GluR2 expression levels compared with untreated control sister cultures (Supplemental Fig. S10).

We then infected slices with lentivirus expressing OPHN1\#2 shRNA at $1 \mathrm{DIV}$, incubated them with the GluR2-3Y peptide from 4 DIV, and performed simultaneous recordings of eEPSCs from a CA1 pyramidal neuron expressing OPHN1\#2 shRNA and an adjacent noninfected control neuron at 8 DIV. Strikingly, incubation with the GluR2-3Y peptide for 4 d completely prevented the effect of OPHN1 RNAi on AMPAR-mediated transmission (Fig. 7D), and to some extent diminished the effect of OPHN1 RNAi on NMDAR-mediated transmission (Fig. 7D). Incubation of slices with the control GluR2-3A peptide, which has no effect on AMPAR stabilization, had no effect on OPHN1\#2 shRNA-induced synaptic depression (Fig. 7E), suggesting that the effects of GluR2-3Y were specific.

Next, we examined whether incubation of slices with GluR2-3Y peptide could also prevent the effect of OPHN1 RNAi on spine density and size observed at 8 DIV. Interestingly, incubation of slices with GluR2-3Y peptide abolished the effect of OPHN1\#2 shRNA on both spine density and spine size (Fig. 7F,H). Given that the GluR2$3 \mathrm{Y}$ peptide was applied at $4 \mathrm{DIV}$, when synapses are still made (see above), these data imply that keeping AMPARs at synapses prevents the destabilization of spine structure caused by extended knockdown of OPHN1.

To further assess the involvement of OPHN1 in AMPAR stabilization, we examined whether ectopic OPHN1 expression influences NMDA-stimulated endocytosis of the AMPAR GluR2 subunit. Application of exogenous NMDA to hippocampal neurons has previously been shown to cause rapid endocytosis of GluR2 (Braithwaite et al. 2002). To quantify the degree of NMDA-induced internalization of endogenous GluR2, we used a biochemical method to cross-link surface-only GluR2 (Hall and Soderling 1997). Hippocampal neurons expressing OPHN1-WT and EGFP, or EGFP alone, were left untreated or treated with NMDA, and subsequently incubated with the membrane-impermeant cross-linking reagent bis (sulfosuccinimidyl) suberate $\left(\mathrm{BS}^{3}\right)$. Western blotting with an anti-GluR2 antibody revealed that NMDA treatment of control EGFP-expressing neurons, as expected, decreased cell-surface GluR2 expression and increased internalized GuR2 levels (Fig. 8A,B). The NMDA-induced decrease in cell-surface GluR2 expression and increase in internal GluR2 levels were significantly attenuated in neurons ectopically expressing OPHN1-WT. Notably, these neurons in general displayed relatively higher basal GluR2 surface levels than the control neurons. These data imply that expression of OPHN1 stabilizes AMPARs in the plasma membrane. Since OPHN1 represses the RhoA/Rho-kinase pathway in hippocampal neurons (Supplemental Figs. S3, S4; Govek et al. 2004), we next examined the effect of RhoA/Rho-kinase inhibition on NMDA-induced GluR2 endocytosis. We found that, akin to ectopic OPHN1 expression, treatment of neurons with the Rho-kinase inhibitor, Y-27632, significantly attenuated the NMDAinduced decrease in cell surface GluR2 expression and increase in internal GluR2 levels (Fig. 8C,D). Thus, these data are consistent with the idea that OPHN1-at least in part by suppressing the RhoA/Rho-kinase pathwaystabilizes a population of AMPARs in the synapse and makes them resistant to regulated endocytosis.

Collectively, our results indicate that OPHN1-mediated signaling controls synaptic function and structure by regulating AMPAR stabilization in the synapse.

\section{Discussion}

Mutations in the OPHN1 gene have been shown to be the cause of X-linked MR associated with cerebellar hypoplasia and enlargement of the ventricles (Tentler et al. 1999; Bergmann et al. 2003; Philip et al. 2003; des Portes 
Figure 7. OPHN1 regulates synaptic function and structure by stabilizing AMPA receptors. $(A)$ Representative traces of excitatory miniature events recorded at $-60 \mathrm{mV}$ from untreated neurons (control) and neurons treated for $4 \mathrm{~d}$ with GluR2-3A or GluR2-3Y. Bars, $5 \mathrm{sec}$ and 20 pA. $(B, C)$ Quantification of mEPSC frequency $(B)$ and amplitude $(C)$ for control, GluR2-3A-treated, and GluR2-3Y-treated neurons (control: $n=17$ cells; GluR23A: $n=16$ cells; GluR2-3Y: $n=22$ cells). $\left(^{\star \star}\right) P<0.01$ by Student's $t$-test. ( $D, E$, top panels) Representative traces of EPSCs recorded simultaneously from a pair of uninfected neurons (control) and OPHN1\#2 shRNA-expressing neurons treated with GluR2-3Y peptide $(D)$ or GluR2-3A peptide (E). Bars, $20 \mathrm{msec}$ and $20 \mathrm{pA}$. (Bottom panel) Quantification of the EPSCs mediated by both AMPAR and NMDAR from pairs of neurons treated with GluR23Y or GluR2-3A peptide. (D) GluR2-3Y: $n=15$ pairs. $(E)$ GluR2-3A: $n=9$ pairs. $(F)$ Representative TPLSM images of secondary apical dendrites from CA1 pyramidal neurons expressing EGFP only (control) or OPHN1\#2 shRNA and EGFP (OPHN1\#2) treated with GluR2-3Y peptide. Bar, $10 \mu \mathrm{m} .(G, H)$ Quantification of spine density $(G)$ and spine size $(H)$ for control and OPHN1\#2 shRNA-expressing neurons treated with GluR2-3Y peptide $(n=4$ cells for both groups). Data are shown as mean \pm SEM. $\left(^{\star}\right) P<0.05$; $\left(^{\star \star}\right)$ $P<0.01$ by paired Student's $t$-test.
A

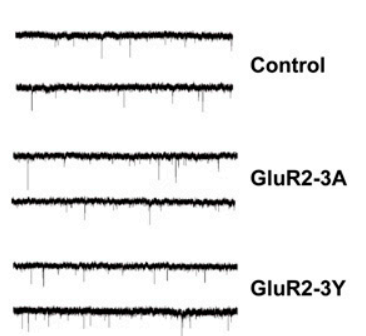

L

D

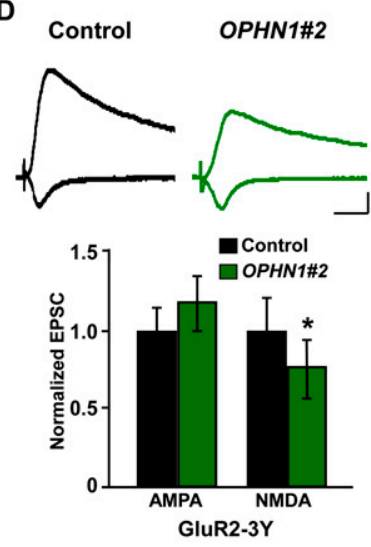

$F$

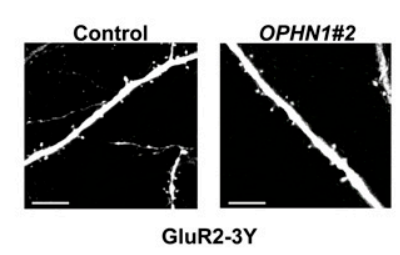

B

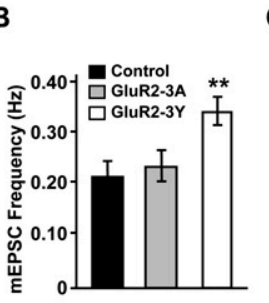

C

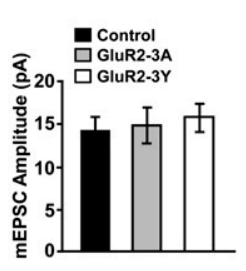

E

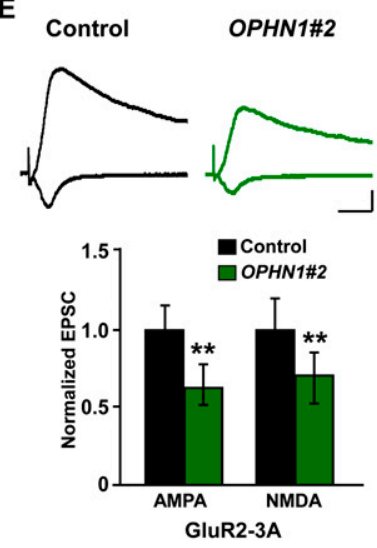

G

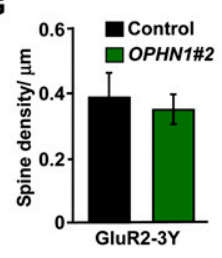

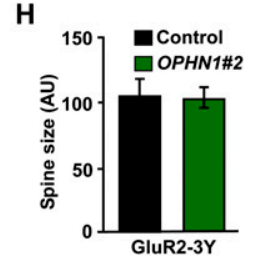

et al. 2004; Zanni et al. 2005). Thus far, however, the underlying pathophysiology of cognitive dysfunction resulting from mutations in OPHN1 remains largely elusive. Our study provides a link between genetic deficits in OPHN1, which are associated with OPHN1 loss of function, and glutamatergic dysfunction.

By temporally and spatially manipulating OPHN1 gene expression at the hippocampal CA3-CA1 pathway, we demonstrate that post-synaptic OPHN1 controls activitydependent maturation and plasticity of excitatory synaptic structure and function by regulating AMPAR stability. In particular, OPHN1 is recruited or stabilized in dendritic spines by spontaneous activity through the activation of NMDARs. In turn, OPHN1 signaling regulates activity-dependent AMPAR synaptic incorporation and stabilization, as well as maintenance of spine structure, thereby permitting synaptic maturation and plasticity. Decreased or defective OPHN1 signaling therefore causes destabilization of synaptic AMPARs and spine structure, leading to impairment in plasticity and eventually loss of spines/synapses and NMDARs. In view of these findings, impaired OPHN1 function is likely to impact excitatory circuit development by interfering with normal functional and structural synaptic maturation. Thus, our findings connect genetic perturbation of OPHN1 func- tion to glutamatergic hypofunction and suggest that defects in early circuitry development are an important contributory factor to MR associated with OPHN1 mutations. Since mutations in other genes coding for regulators or effectors of Rho GTPases (including MEGAP, OCRL1, ARHGEF6, and PAK3) have been associated with MR (Nadif Kasri and Van Aelst 2008), it will be interesting to see whether they lead to a common dysfunction at the level of synapse and/or circuitry development. The role, however, of most of these Rho-linked MR proteins in synaptic function, plasticity, and development remains largely unexplored (Endris et al. 2002; Lowe 2005; NodeLanglois et al. 2006; but see Boda et al. 2004; Meng et al. 2005).

Our results unveil an interesting positive feedback relationship between OPHN1 signaling and synaptic activity, in that synaptic activity is required for OPHN1 signaling, and, in turn, OPHN1-mediated signaling enhances the efficacy of synaptic function. Such a positive feedback relationship could play a key role during critical periods of synapse development, with too little activity leading to weakening of the synapse and threshold levels of activity leading to its saturation. Clearly, other signaling events besides those mediated by OPHN1 will come into play to keep synaptic strength within a useful 
A

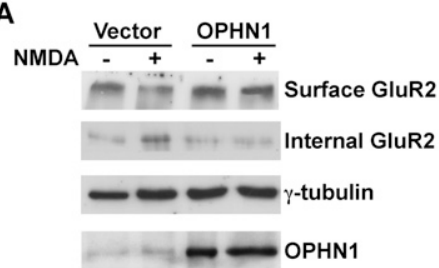

C

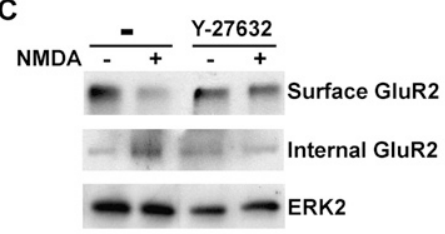

B

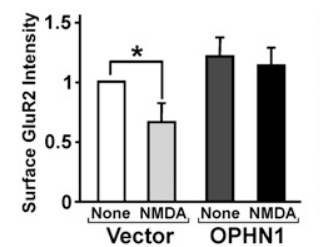

D

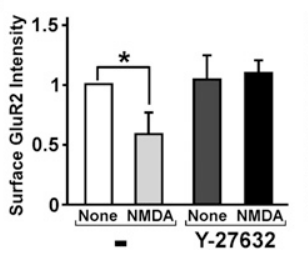

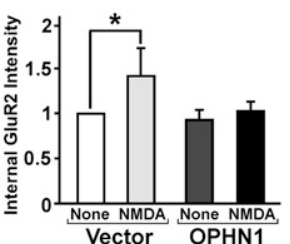

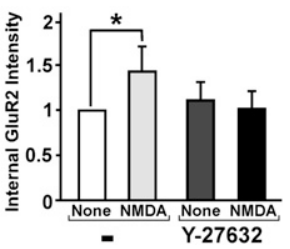

Figure 8. NMDA-induced endocytosis of GluR2 is reduced in neurons ectopically expressing OPHN1 and treated with Rhokinase inhibitor. (A) Surface and internal GluR2 levels were determined by the $\mathrm{BS}^{3}$ cross-linking method. Hippocampal neurons infected with lentiviral vector expressing OPHN1-WT together with EGFP, or EGFP alone, were left untreated $(-)$ or were treated $(+)$ with NMDA and subsequently incubated with $\mathrm{BS}^{3}$ (see the Supplemental Material). Cell extracts were prepared and analyzed with anti-GluR2, anti-OPHN1, and anti- $\gamma$ tubulin as a loading control. $(B)$ Quantification of surface and internal GluR2 levels normalized to those of $\gamma$-tubulin and then normalized to the value of 1 for untreated control vector-expressing cells. Data are shown as mean \pm SEM, $n=3$ independent experiments; $\left(^{\star}\right) P<0.05 .(C, D)$ Same as $A$ and $B$, except that uninfected hippocampal neurons were used that were left untreated $(-)$ or treated $(+)$ with NMDA in the absence or presence of the Rho-kinase inhibitor Y-27632 $(10 \mu \mathrm{M})$, and data were normalized to those of ERK2 and then normalized to the value of 1 for untreated cells.

dynamic range. The coupling between the function of OPHN1 in excitatory synapses and synaptic activity also suggests that the well-documented impact of experience on synaptic function and structure is likely to involve OPHN1-mediated signaling.

An important finding of this study is that activitydependent OPHN1 signaling controls synaptic structure and function by regulating AMPAR stability, and that the underlying mechanism involves OPHN1's Rho-GAP activity and a RhoA/Rho-kinase-mediated signaling pathway. Indeed, we found that stabilizing AMPARs in the synapse prevents both the defects in spine structure and AMPAR-mediated transmission caused by knockdown of OPHN1, and that, akin to ectopic expression of OPHN1WT, inhibition of the RhoA/Rho-kinase pathway attenuates NMDA-stimulated AMPAR endocytosis. Moreover, we observed that OPHN1's Rho-GAP activity is important for maintaining spine structure, potentiating AMPAR-mediated transmission, and structural and functional plasticity.

In general, the biological functions of Rho-GAPs are dependent on their subcellular localization (Govek et al. 2005); a local increase in their concentration and/or activity upon an extracellular stimulus or cue can thereby regulate the activity of Rho GTPases in a spatio-temporal manner. We found that synaptic activity through NMDAR activation drives OPHN1 into dendritic spines, where it forms a complex with AMPARs. Therefore, the local increase in OPHN1 likely modulates RhoA and Rhokinase activities in the proximity of AMPARs. In this regard, previous studies have demonstrated a decrease in endogenous RhoA activity upon glutamate receptor activation (Van Aelst and Cline 2004; Kang et al. 2009). As mentioned before, a main target of RhoA/Rho-kinase is the actin cytoskeleton, and nascent actin filaments have been implicated in the stabilization of AMPARs (for review, see Cingolani and Goda 2008). Thus, a likely scenario is that spine enriched OPHN1 by locally modulating RhoA/Rhokinase activities and actin dynamics (i.e., in the proximity of AMPARs) contributes to the stabilization of AMPARs. Given that actin remodeling has been suggested to be required, but not sufficient on its own, to potentiate synaptic transmission (Kim and Lisman 1999; Krucker et al. 2000; Fukazawa et al. 2003), we do not exclude, however, that actin-independent functions of the OPHN1/ RhoA pathway could also be involved in mediating OPHN1 function. One such possibility could be a particular RhoA signaling pathway that regulates endocytic trafficking (Govek et al. 2005). Also, OPHN1 through interaction with AMPARs could potentially contribute to the stabilization of AMPARs; this interaction, however, is likely to involve other partners and/or post-translational modifications.

In this study, we selectively manipulated OPHN1 levels in individual CA1 neurons in hippocampal slices during a critical period of synapse development in order to examine the function of OPHN1 in a cell-autonomous manner. This is important because OPHN1 is expressed both post- and presynaptically (Govek et al. 2004). In fact, this could be one of the reasons why Khelfaoui et al. (2007) failed to monitor a change in basal excitatory transmission in the CA1 hippocampus of adult ophn1 global knockout mice; although, surprisingly, they did observe a reduction in the number of mature spines. Another complication is that they compared field EPSPs across brain slices, without measuring input output curves or measuring miniature EPSCs, which makes it difficult to compare differences in basal transmission between wild-type and ophn1 knockout mice. In our study, comparisons of synaptic transmission between wild-type neurons and neurons expressing OPHN1 shRNAs were performed simultaneously within the same slice. Of note, Khelfaoui et al. (2007) did report a decrease in PPF, a measurement of presynaptic release in the OPHN1 knockout mice. We found that selective knockdown of OPHN1 at the post-synaptic site does not affect PPF, suggesting that the impaired PPF they described is likely to result from presynaptic loss of 
OPHN1 function, or alternatively is due to a compensatory adaptation to the post-synaptic defect caused by very long-term loss of OPHN1 in ophn1 knockout mice. Experiments designed to selectively interfere with OPHN1 function at the presynaptic site will be needed to elucidate this.

In summary, our results reveal that activity-dependent OPHN1-mediated signaling controls the stabilization of AMPARs at the synapse and thus permits synaptic plasticity and synaptic maturation. Therefore, loss of OPHN1 (as is the case in individuals carrying OPHN1 mutations) leads to impaired synaptic development and decreased glutamatergic function. Thus, our study provides a mechanism by which genetic deficits in OPHN1 that are associated with MR are linked to developmental abnormalities and glutamatergic hypofunction. It further highlights the concept that hypofunction of the glutamatergic system within specific circuits can contribute to cognitive and neurodevelopmental disorders (Hsieh et al. 2006; Chahrour and Zoghbi 2007; Li et al. 2007). It should be noted that also presynaptic loss of OPHN1 could contribute to the pathogenesis of OPHN1-linked cognitive impairment, as OPHN1 is present both post- and presynaptically. The future elucidation of OPHN1's presynaptic function should shed further light on this.

\section{Materials and methods}

Hippocampal slice cultures, lentiviral infection, transfection, and other treatments

Organotypic hippocampal slice cultures were prepared from postnatal day 6 or 7 rat pups as described (Govek et al. 2004). Slices were infected after $1 \mathrm{~d}$ in culture $(1 \mathrm{DIV})$ and used for electrophysiology or imaging experiments at 4 or 8 DIV as indicated. To infect hippocampal slice cultures, concentrated viral solution was injected into the CAl pyramidal cell layer using a Picospritzer II (General Valve). For OPHN1 localization, time-lapse imaging, and cLTP experiments, slices were biolistically transfected at 7 DIV using a Helios Gene Gun (Bio-Rad) and imaged 4 and/or $8 \mathrm{~d}$ post-transfection.

For treatment with TTX, APV, high $\mathrm{MgCl}_{2}$ concentration, or GluR2-3Y or 3A peptides, slices were infected with lentivirus at day 1. At day 4, TTX $(1 \mu \mathrm{M})$, APV $(100 \mu \mathrm{M}), \mathrm{MgCl}_{2}(10 \mathrm{mM})$ or the peptides $(5 \mu \mathrm{M})$ were added to the medium. TTX, APV, or peptides were replenished every $24 \mathrm{~h}$. Recordings were made on day 8 . The sequences of GluR2-3Y and $3 \mathrm{~A}$ are the same as previously reported (Ahmadian et al. 2004). A TAT sequence was fused to peptides to aid delivery into the cell. A dansyl group was also included for visualization of the peptides (Li et al. 2007). Accumulation of the peptides in neurons was readily detectable by TPLSM at a wavelength of $720 \mathrm{~nm}$ (data not shown).

\section{Electrophysiology}

Whole-cell recordings were obtained with Axopatch-1D or Multiclamp 700B amplifiers (Axon Instruments). To monitor OPHN1's effects on synaptic transmission, slices were infected with the respective indicated lentiviruses. Four days to $8 \mathrm{~d}$ later, whole-cell recordings were obtained simultaneously from an infected and an adjacent uninfected neuron in the CAl region under visual guidance using epifluorescence and transmitted light illumination. The recording chamber was perfused with artificial cerebrospinal fluid (ACSF) containing $119 \mathrm{mM} \mathrm{NaCl}$, $2.5 \mathrm{mM} \mathrm{KCl}_{1} 4 \mathrm{mM} \mathrm{CaCl}_{2}, 4 \mathrm{mM} \mathrm{MgCl}_{2}, 26 \mathrm{mM} \mathrm{NaHCO}_{3}, 1 \mathrm{mM}$ $\mathrm{NaH}_{2} \mathrm{PO}_{4}, 11 \mathrm{mM}$ glucose, $0.1 \mathrm{mM}$ picrotoxin, and $4 \mu \mathrm{M}$ 2-chloroadenosine $(\mathrm{pH} 7.4)$, and gassed with $5 \% \mathrm{CO}_{2} / 95 \% \mathrm{O}_{2}$. Recordings were made at $27^{\circ} \mathrm{C}$. Patch recording pipettes $(3-5$ $\mathrm{M} \Omega$ ) were filled with intracellular solution containing $115 \mathrm{mM}$ cesium methanesulfonate, $20 \mathrm{mM} \mathrm{CsCl}, 10 \mathrm{mM}$ HEPES, $2.5 \mathrm{mM}$ $\mathrm{MgCl}_{2}, 4 \mathrm{mM} \mathrm{Na} \mathrm{mTP}_{2} \mathrm{ATP}$ mM Na $\mathrm{mTP}_{3}, 10 \mathrm{mM}$ sodium phosphocreatine, and 0.6 mM EGTA (pH 7.25). Evoked responses were induced using bipolar electrodes placed on Schaffer collateral pathway. Responses were recorded at both $-60 \mathrm{mV}$ (for AMPAR-mediated responses) and $+40 \mathrm{mV}$ (for NMDAR-mediated responses). NMDAR-mediated responses were quantified as the mean between 60 and $65 \mathrm{msec}$ after stimulation. All recordings were done by stimulating two independent synaptic inputs; results from each pathway were averaged and counted as $n=1$.

Conventional LTP was induced by pairing presynaptic stimulation at $3 \mathrm{~Hz}$ with depolarization of the post-synaptic neuron at $0 \mathrm{mV}$ for $3 \mathrm{~min}$; recordings were maintained for at least $40 \mathrm{~min}$ after pairing. The EPSC amplitude was normalized to the average baseline amplitude before pairing.

Spontaneous responses were recorded at $-60 \mathrm{mV}$ (mEPSC) and $+10 \mathrm{mV}$ (mIPSC) in ACSF containing $2.5 \mathrm{mM} \mathrm{CaCl}_{2}$ and $1.2 \mathrm{mM}$ $\mathrm{MgCl}_{2}$ at $27^{\circ} \mathrm{C}$. mEPSCs were recorded in the presence of $1 \mu \mathrm{M}$ TTX and $0.1 \mathrm{mM}$ picrotoxin, whereas mIPSCs were recorded in the presence of $10 \mu \mathrm{M}$ CNQX and $100 \mu \mathrm{M}$ APV. Five to $10 \mathrm{~min}$ of recordings were analyzed from each cell. Data were acquired at 5 $\mathrm{kHz}$, filtered at $2 \mathrm{kHz}$, and analyzed using the Mini Analysis Program (Synaptosoft). All data are reported as mean \pm SEM. Statistical significance was determined by the paired Student's $t$ test (for paired recordings) or the Mann-Whitney test (for unpaired recordings). Significance was set to $P<0.05$.

TPLSM and CLTP induction, image display, and quantitative image analysis

These procedures were conducted largely as described in Kopec et al. (2006); and see the Supplemental Material.

\section{Other procedures}

Generation of cDNA and shRNA constructs and methods for immunofluorescence, transfection and infection of dissociated hippocampal cultures, preparation of synaptosomes, coimmunoprecipitation, RhoA activation, and AMPAR surface labeling are described in the Supplemental Material.

\section{Acknowledgments}

We thank Dr. E.E. Govek and members of the Van Aelst and Malinow laboratory for discussions and/or critical reading of the manuscript, and N. Dawkins and Dr. W. Shen for technical assistance. This work was supported by NSF and NIH grants to L.V.A. N.N.K. is a post-doctoral fellow from the Fund for Scientific Research Flanders and is supported by the Human Frontiers Science Program.

\section{References}

Ahmadian G, Ju W, Liu L, Wyszynski M, Lee SH, Dunah AW, Taghibiglou C, Wang Y, Lu J, Wong TP, et al. 2004. Tyrosine phosphorylation of GluR2 is required for insulin-stimulated AMPA receptor endocytosis and LTD. EMBO J 23: 1040-1050.

Bagni C, Greenough WT. 2005. From mRNP trafficking to spine dysmorphogenesis: The roots of fragile X syndrome. Nat ReV Neurosci 6: 376-387. 
Barria A, Malinow R. 2005. NMDA receptor subunit composition controls synaptic plasticity by regulating binding to CaMKII. Neuron 48: 289-301.

Bergmann C, Zerres K, Senderek J, Rudnik-Schoneborn S, Eggermann T, Hausler M, Mull M, Ramaekers VT. 2003. Oligophrenin 1 (OPHN1) gene mutation causes syndromic $\mathrm{X}$-linked mental retardation with epilepsy, rostral ventricular enlargement and cerebellar hypoplasia. Brain 126: 15371544.

Bienvenu T, Der-Sarkissian H, Billuart P, Tissot M, Des Portes V, Bruls T, Chabrolle JP, Chauveau P, Cherry M, Kahn A, et al. 1997. Mapping of the X-breakpoint involved in a balanced $\mathrm{X}_{;} 12$ translocation in a female with mild mental retardation. Eur I Hum Genet 5: 105-109.

Billuart P, Bienvenu T, Ronce N, des Portes V, Vinet MC, Zemni R, Roest Crollius H, Carrie A, Fauchereau F, Cherry M, et al. 1998. Oligophrenin-1 encodes a rhoGAP protein involved in $\mathrm{X}$-linked mental retardation. Nature 392: 923-926.

Boda B, Alberi S, Nikonenko I, Node-Langlois R, Jourdain P, Moosmayer M, Parisi-Jourdain L, Muller D. 2004. The mental retardation protein PAK3 contributes to synapse formation and plasticity in hippocampus. J Neurosci 24: 1081610825.

Braithwaite SP, Xia H, Malenka RC. 2002. Differential roles for NSF and GRIP/ABP in AMPA receptor cycling. Proc Natl Acad Sci 99: 7096-7101.

Brebner K, Wong TP, Liu L, Liu Y, Campsall P, Gray S, Phelps L, Phillips AG, Wang YT. 2005. Nucleus accumbens long-term depression and the expression of behavioral sensitization. Science 310: 1340-1343.

Chahrour M, Zoghbi HY. 2007. The story of Rett syndrome: From clinic to neurobiology. Neuron 56: 422-437.

Chelly J, Khelfaoui M, Francis F, Cherif B, Bienvenu T. 2006. Genetics and pathophysiology of mental retardation. Eur $I$ Hum Genet 14: 701-713.

Cingolani LA, Goda Y. 2008. Actin in action: The interplay between the actin cytoskeleton and synaptic efficacy. Nat Rev Neurosci 9: 344-356.

Citri A, Malenka RC. 2008. Synaptic plasticity: Multiple forms, functions, and mechanisms. Neuropsychopharmacology 33: $18-41$.

des Portes V, Boddaert N, Sacco S, Briault S, Maincent K, Bahi N, Gomot M, Ronce N, Bursztyn J, Adamsbaum C, et al. 2004. Specific clinical and brain MRI features in mentally retarded patients with mutations in the Oligophrenin-1 gene. Am I Med Genet A 124: 364-371.

Endris V, Wogatzky B, Leimer U, Bartsch D, Zatyka M, Latif F, Maher ER, Tariverdian G, Kirsch S, Karch D, et al. 2002. The novel Rho-GTPase activating gene MEGAP/ srGAP3 has a putative role in severe mental retardation. Proc Natl Acad Sci 99: 11754-11759.

Engert F, Bonhoeffer T. 1999. Dendritic spine changes associated with hippocampal long-term synaptic plasticity. Nature 399: 66-70.

Fox CJ, Russell K, Titterness AK, Wang YT, Christie BR. 2007. Tyrosine phosphorylation of the GluR2 subunit is required for long-term depression of synaptic efficacy in young animals in vivo. Hippocampus 17: 600-605.

Fukazawa Y, Saitoh Y, Ozawa F, Ohta Y, Mizuno K, Inokuchi K. 2003. Hippocampal LTP is accompanied by enhanced F-actin content within the dendritic spine that is essential for late LTP maintenance in vivo. Neuron 38: 447-460.

Govek EE, Newey SE, Akerman CJ, Cross JR, Van der Veken L, Van Aelst L. 2004. The X-linked mental retardation protein oligophrenin-1 is required for dendritic spine morphogenesis. Nat Neurosci 7: 364-372.
Govek EE, Newey SE, Van Aelst L. 2005. The role of the Rho GTPases in neuronal development. Genes \& Dev 19: 1-49.

Hall RA, Soderling TR. 1997. Quantitation of AMPA receptor surface expression in cultured hippocampal neurons. Neuroscience 78: 361-371.

Harris KM, Jensen FE, Tsao B. 1992. Three-dimensional structure of dendritic spines and synapses in rat hippocampus (CA1) at postnatal day 15 and adult ages: Implications for the maturation of synaptic physiology and long-term potentiation. J Neurosci 12: 2685-2705.

Hsieh H, Boehm J, Sato C, Iwatsubo T, Tomita T, Sisodia S, Malinow R. 2006. AMPAR removal underlies A $\beta$-induced synaptic depression and dendritic spine loss. Neuron 52: 831843.

Inlow JK, Restifo LL. 2004. Molecular and comparative genetics of mental retardation. Genetics 166: 835-881.

Kang MG, Guo Y, Huganir RL. 2009. AMPA receptor and GEFH1/Lfc complex regulates dendritic spine development through RhoA signaling cascade. Proc Natl Acad Sci 106: 3549-3554.

Khelfaoui M, Denis C, van Galen E, de Bock F, Schmitt A, Houbron C, Morice E, Giros B, Ramakers G, Fagni L, et al. 2007. Loss of X-linked mental retardation gene oligophrenin 1 in mice impairs spatial memory and leads to ventricular enlargement and dendritic spine immaturity. J Neurosci 27: 9439-9450.

Kim CH, Lisman JE. 1999. A role of actin filament in synaptic transmission and long-term potentiation. I Neurosci 19: 4314-4324.

Kopec CD, Li B, Wei W, Boehm J, Malinow R. 2006. Glutamate receptor exocytosis and spine enlargement during chemically induced long-term potentiation. J Neurosci 26: 2000-2009.

Kopec CD, Real E, Kessels HW, Malinow R. 2007. GluR1 links structural and functional plasticity at excitatory synapses. I Neurosci 27: 13706-13718.

Krucker T, Siggins GR, Halpain S. 2000. Dynamic actin filaments are required for stable long-term potentiation (LTP) in area CA1 of the hippocampus. Proc Natl Acad Sci 97: 68566861.

Li Z, Sheng M. 2003. Some assembly required: The development of neuronal synapses. Nat Rev Mol Cell Biol 4: 833-841.

Li B, Woo RS, Mei L, Malinow R. 2007. The neuregulin-1 receptor erbB4 controls glutamatergic synapse maturation and plasticity. Neuron 54: 583-597.

Lowe M. 2005. Structure and function of the Lowe syndrome protein OCRL1. Traffic 6: 711-719.

Matsuzaki M, Honkura N, Ellis-Davies GC, Kasai H. 2004. Structural basis of long-term potentiation in single dendritic spines. Nature 429: 761-766.

McAllister AK. 2007. Dynamic aspects of CNS synapse formation. Annu Rev Neurosci 30: 425-450.

Meng J, Meng Y, Hanna A, Janus C, Jia Z. 2005. Abnormal longlasting synaptic plasticity and cognition in mice lacking the mental retardation gene Pak3. J Neurosci 25: 6641-6650.

Nadif Kasri N, Van Aelst L. 2008. Rho-linked genes and neurological disorders. Pflugers Arch 455: 787-797.

Nakayama AY, Harms MB, Luo L. 2000. Small GTPases Rac and Rho in the maintenance of dendritic spines and branches in hippocampal pyramidal neurons. J Neurosci 20: 5329-5338.

Node-Langlois R, Muller D, Boda B. 2006. Sequential implication of the mental retardation proteins ARHGEF6 and PAK3 in spine morphogenesis. J Cell Sci 119: 4986-4993.

Philip N, Chabrol B, Lossi AM, Cardoso C, Guerrini R, Dobyns WB, Raybaud C, Villard L. 2003. Mutations in the oligophrenin-1 gene (OPHN1) cause X linked congenital cerebellar hypoplasia. J Med Genet 40: 441-446. 
Ryan XP, Alldritt J, Svenningsson P, Allen PB, Wu GY, Nairn AC, Greengard P. 2005. The Rho-specific GEF Lfc interacts with neurabin and spinophilin to regulate dendritic spine morphology. Neuron 47: 85-100.

Sfakianos MK, Eisman A, Gourley SL, Bradley WD, Scheetz AJ, Settleman J, Taylor JR, Greer CA, Williamson A, Koleske AJ. 2007. Inhibition of Rho via Arg and p190RhoGAP in the postnatal mouse hippocampus regulates dendritic spine maturation, synapse and dendrite stability, and behavior. $J$ Neurosci 27: 10982-10992.

Shepherd JD, Huganir RL. 2007. The cell biology of synaptic plasticity: AMPA receptor trafficking. Annu Rev Cell Dev Biol 23: 613-643.

Tashiro A, Minden A, Yuste R. 2000. Regulation of dendritic spine morphology by the rho family of small GTPases: Antagonistic roles of Rac and Rho. Cereb Cortex 10: 927-938.

Tentler D, Gustavsson P, Leisti J, Schueler M, Chelly J, Timonen E, Anneren G, Willard HF, Dahl N. 1999. Deletion including the oligophrenin-1 gene associated with enlarged cerebral ventricles, cerebellar hypoplasia, seizures and ataxia. Eur $J$ Hum Genet 7: 541-548.

Vaillend C, Poirier R, Laroche S. 2008. Genes, plasticity and mental retardation. Behav Brain Res 192: 88-105.

Van Aelst L, Cline HT. 2004. Rho GTPases and activitydependent dendrite development. Curr Opin Neurobiol 14: 297-304.

van Galen EJ, Ramakers GJ. 2005. Rho proteins, mental retardation and the neurobiological basis of intelligence. Prog Brain Res 147: 295-317.

Wong TP, Howland JG, Robillard JM, Ge Y, Yu W, Titterness AK, Brebner K, Liu L, Weinberg J, Christie BR, et al. 2007. Hippocampal long-term depression mediates acute stressinduced spatial memory retrieval impairment. Proc Natl Acad Sci 104: 11471-11476.

Zanni G, Saillour Y, Nagara M, Billuart P, Castelnau L, Moraine C, Faivre L, Bertini E, Durr A, Guichet A, et al. 2005. Oligophrenin 1 mutations frequently cause X-linked mental retardation with cerebellar hypoplasia. Neurology 65: 13641369.

Zhu JJ, Qin Y, Zhao M, Van Aelst L, Malinow R. 2002. Ras and Rap control AMPA receptor trafficking during synaptic plasticity. Cell 110: 443-455. 


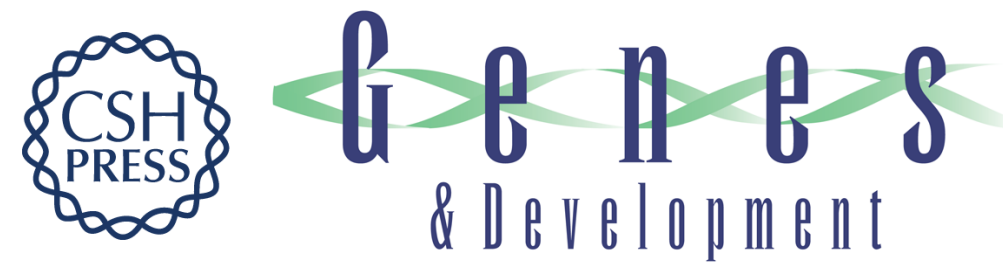

\section{The Rho-linked mental retardation protein oligophrenin-1 controls synapse maturation and plasticity by stabilizing AMPA receptors}

Nael Nadif Kasri, Akiko Nakano-Kobayashi, Roberto Malinow, et al.

Genes Dev. 2009, 23:

Access the most recent version at doi:10.1101/gad.1783809

Supplemental http://genesdev.cshlp.org/content/suppl/2009/05/20/23.11.1289.DC1
Material

References This article cites 51 articles, 20 of which can be accessed free at:

http://genesdev.cshlp.org/content/23/11/1289.full.html\#ref-list-1

License

Email Alerting Receive free email alerts when new articles cite this article - sign up in the box at the top

Service

right corner of the article or click here.

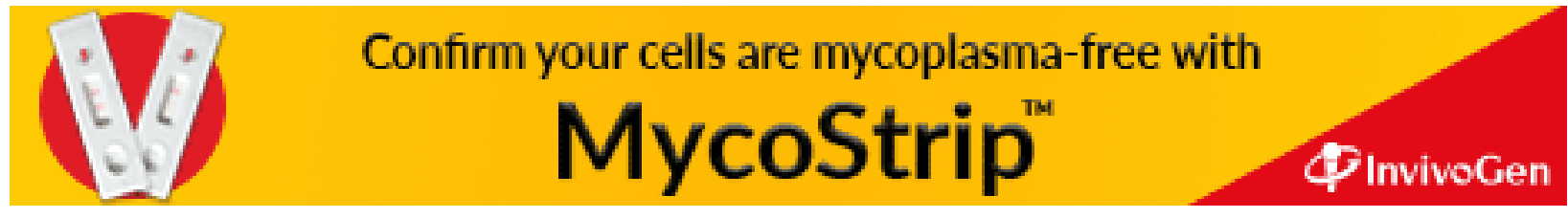

